\title{
Patterns of GRP78 and MTJ1 expression in primary cutaneous malignant melanoma
}

\author{
John A Papalas, Robin T Vollmer, Mario Gonzalez-Gronow, Salvatore V Pizzo, \\ James Burchette, Kenneth E Youens, Krystal B Johnson and Maria A Selim
}

Department of Pathology, Duke University Medical Center, Durham, NC, USA

\begin{abstract}
Cell surface expression of glucose-regulated protein 78 (GRP78) occurs in several types of cancer; however, its role in the behavior of primary cutaneous melanoma is not well studied. The association of cell surface GRP78 with other proteins such as MTJ1 stimulates cell proliferation. In this study, we characterized the pattern of expression of GRP78 and MTJ1 in invasive primary cutaneous melanomas and analyzed the relationships between the pattern of expression and various clinicopathological parameters. We found two patterns of GRP78 expression in invasive primary cutaneous melanoma. One pattern showed a gradual fading of protein expression from superficial to deeper levels within the same tumor. The second pattern of expression showed a similar fading with an abrupt regaining of expression at the deep invasive edge of the melanoma. These two distinct patterns of GRP78 expression correlated with both patient survival and depth of tumor invasion. A moderate MTJ1 expression was found to be associated with decreased patient survival; however, no significant associations were observed between patterns of GRP78 and MTJ1 expression. Our study (1) describes two distinct patterns of GRP78 in invasive primary cutaneous melanoma, (2) inversely correlates regain of GRP78 expression with patient survival, and (3) suggests a modifying effect of MTJ1 on GRP78 in enhancing tumor aggressiveness.
\end{abstract}

Modern Pathology (2010) 23, 134-143; doi:10.1038/modpathol.2009.152; published online 16 October 2009

Keywords: GRP78; MTJ1; melanoma; heat shock protein; chaperone protein

The incidence of malignant melanoma in the United States continues to rise. ${ }^{1}$ There has, however, been a concomitant decrease in mortality, which, at least for some melanoma subtypes, is linked to a proportionately earlier declaration of tumors at smaller Breslow thicknesses. ${ }^{2}$ Today, melanoma therapeutic and diagnostic research models are based largely on genetic and molecular studies of melanoma pathogenesis. ${ }^{3-5}$ Within this broad field, the study of the physiological and transcriptional differences between more superficial and deeper melanoma cells has emerged as an active area of research. ${ }^{6-8}$ Our study aims to contribute to the understanding of intralesional differences in protein expression by malignant cells.

Glucose-regulated protein 78 (GRP78) is a resident endoplasmic reticulum (ER) chaperone protein first

Correspondence: Dr JA Papalas, MD, Department of Pathology, Duke University Medical Center, Box 3712, Durham, NC 27710, USA.

E-mail: papal002@mc.duke.edu

Received 14 April 2009; revised and accepted 14 August 2009; published online 16 October 2009 described in $1977 .^{9}$ As a family, GRPs are upregulated in glucose-starved cell cultures and in other stress-induced states. ${ }^{10}$ As part of the ER proteinfolding machinery, the critical role of GRP78 in the unfolded protein response (UPR) has been well characterized. ${ }^{11}$ GRP78 has a major role in regulating intracellular protein trafficking, ${ }^{12}$ apoptosis, ${ }^{13,14}$ cell surface receptor-mediated endocytosis, ${ }^{15}$ and in a variety of cells, as a cell surface protein that functions as a receptor. ${ }^{16}$

As a cell surface protein, GRP78 binds a number of different ligands. One of its functions is to serve as a signaling receptor for activated $\alpha 2$-macroglobulin. ${ }^{17-19}$ Cell surface GRP78 is also a major autoantigen in various cancers. ${ }^{20-22}$ As opposed to its ERbased role in apoptosis and inhibition of protein synthesis, as a cell surface receptor, GRP78 signaling results in a net increase in DNA synthesis, protein synthesis and cellular proliferation. ${ }^{23-25}$ Studies suggest that central to its function in this regard is the cell surface interaction between GRP78 and MTJ1, a DnaJ-like protein..$^{23,26,27}$ As a class, DnaJ-like proteins (such as MTJ1) generally assist in protein folding and trafficking. ${ }^{28,29}$ The role of MTJ1 in 
neoplasia has not been extensively characterized and it currently seems that the significance of this DnaJ-like protein resides in its ability to modify cell surface GRP78 signaling as described above. ${ }^{23}$

For over a decade, investigations have explored the divergent functions of GRP78 in nonmelanocytic malignancies, and have begun to focus on targeting the protein for a variety of therapeutic applications. ${ }^{15,30}$ GRP78 expression has been studied in a variety of cancer cell lines, including the breast, melanoma, and prostate. ${ }^{31-34}$ Quantitative analysis of GRP78 shows elevated levels in hepatocellular carcinoma and gastric cancer relative to surrounding nontumor tissue. ${ }^{35,36}$ Currently, correlations have been drawn between various clinicopathological parameters and levels of GRP78 expression in esophageal, ${ }^{37}$ lung, ${ }^{38}$ and prostate cancers, ${ }^{39}$ and most recently, melanoma. ${ }^{40}$

Glucose-regulated protein 78 has only recently begun to be studied in malignant melanoma excised from human subjects. ${ }^{40}$ Although it is known to be expressed in cultured melanoma cell lines ${ }^{41}$ and it binds selectively to antigens isolated from terminally differentiated melanoma cells, ${ }^{42,43}$ investigations into the clinicopathological relationship between GRP78 and melanoma are just beginning to emerge. ${ }^{40}$ In this study, we characterize the pattern of GRP78 and MTJ1 expression in invasive melanomas and correlate the pattern of expression with various clinical parameters.

\section{Materials and methods}

\section{Study Subjects}

From a group of 322 patients with primary cutaneous melanoma, who had (1) the primary cutaneous tumor available in our surgical pathology archives, (2) adequate tissue for immunohistochemical staining on the basis of the quality of tissue preservation and thickness of tissue in the paraffin block, and (3) adequate clinical records, we randomly selected a group of 65 cases, a number that we estimated would be needed to achieve statistical significance in our analysis. Cases (seven) were excluded if there was a blinded, intraobserver (JP and MS) disagreement on GRP78 expression pattern (see immunohistochemical interpretation section below). Our patient population was skewed to contain a greater proportion of patients with metastatic tumors $(46 \%)$ than would be expected for patients who had metastatic disease on presentation. Only primary cutaneous melanomas with predominant epithelioid histology were included. For this study, we chose not to perform immunohistochemical analysis on desmoplastic tumors or on tumors with a predominant spindle cell morphology on the assumption that studying a phenotypically heterogeneous group of tumors may introduce potentially confounding variability in the patterns of GRP78 expression, which is already known to occur with some proteins between morphologically different melanoma subtypes. ${ }^{44}$ Demographic and clinical information was obtained from hospital records. This study was approved by the DUMC Institutional Review Board.

\section{Immunohistochemical Staining of GRP78 and MTJ}

Formalin-fixed paraffin-embedded tissue sections (4- to $5-\mu \mathrm{m}$ thick) were placed on positively charged microscope slides, allowed to air dry, and subsequently heated for $20 \mathrm{~min}$ at $65-70^{\circ} \mathrm{C}$. After the removal of paraffin, endogenous peroxidase activity was quenched with hydrogen peroxide in methanol and the sections were hydrated to water. Tissue sections were then placed in preheated $1 \times$ Dako Target Retrieval solution, $\mathrm{pH}$ 6.1, and heated for $20 \mathrm{~min}$ in a $100^{\circ} \mathrm{C}$ water bath. After antigen retrieval, the tissue sections and retrieval solution were allowed to cool for $20 \mathrm{~min}$. The slides were washed with several changes of deionized water and placed in Tris-buffered saline. The charged tissue components were treated with $5 \%$ nonimmune horse serum. Excess serum was drained from each slide. An affinity-purified anti-GRP78 goat polyclonal antibody diluted 1:400 (Santa Cruz Biotechnology Santa Cruz, CA, USA, catalog number SC-1050) directed against an epitope in the NH2-terminal domain and a rabbit anti-MTJ1 IgG specific for an epitope in the MTJ1 region comprising amino acids $\mathrm{V}^{110}$ AIYEVLKDDERRQRYDDIL ${ }^{129}$ diluted 1:50 were applied to separate slides and incubated for $1 \mathrm{~h}$. Detection of the bound primary antibody was accomplished by linking with biotinylated horse anti-goat IgG secondary antibody (Vector Laboratories, Burlingame, CA, USA, catalog number BA9500). ABC Elite tertiary reagent (Vector Laboratories) was used to label the attached secondary antibody. Visualization of the bound immune complex was carried out using 3,3'-diaminobenzidine tetrahydrochloride or permanent 3-amino-9-ethylcarbazole. The sections were counter stained with modified Harris hematoxylin, followed by traditional dehydration, clearing, and mounting.

\section{Immunohistochemical Interpretation}

The slides were independently reviewed by pathologists (JP and AS) who were blinded to both clinical data and other interpretations. Cases (seven) were omitted from the study population if there was a disagreement in GRP78 staining pattern between the two grading pathologists (total study $n=58$ ). Omitted cases showed partial tissue deterioration during antigen retrieval. These cases were then stained with MTJ1 and independently reviewed by JP and AS. As opposed to the qualitative differences seen with GRP78 staining, we observed only quantitative differences in MTJ1 staining and graded each case as absent, weak/blush, or strong. The cases 
that had a disagreement (five cases, all between weak and strong) were graded as blush/weak.

\section{Statistical Methods}

To analyze the relationship between the pattern of GRP78 and MTJ1 staining and metastasis, we used a $\chi^{2}$-test. To analyze the relationships between overall survival and several prognostic variables, we used Kaplan-Meier plots and log-rank tests. As only 33 patients were observed to die, only univariate survival analyses could be carried out.

\section{Results}

\section{Patient Characteristics}

The overall patient characteristics and immunohistochemical staining data are summarized in Figure 1. The cases selected consisted of 36 men and 22 women, ranging in age from 29 to 77 years (mean $=54$ ), of which 26 patients had documented
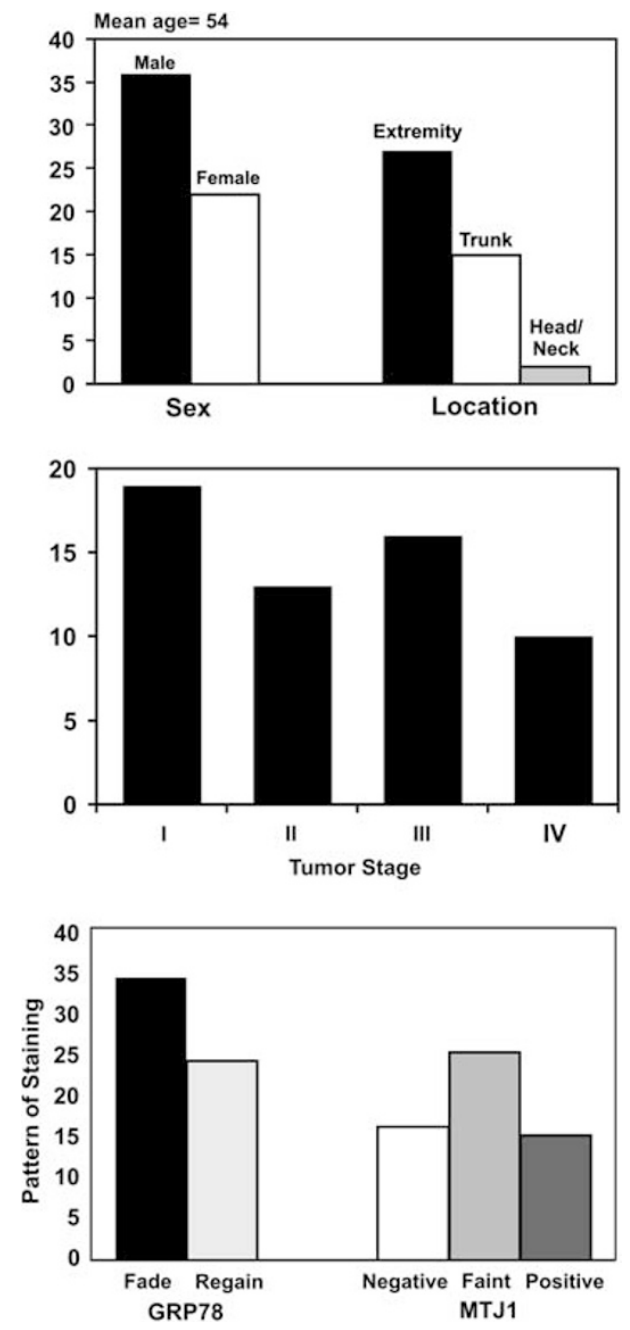

Figure 1 Summary of patient characteristics and staining patterns. metastatic disease at some point throughout their clinical course. A total of 33 patients were followed up until death. The mean (range) of Breslow thickness was $2.8 \mathrm{~mm}(0.6-11 \mathrm{~mm})$. In all, 16 tumors were located in the head and neck, 15 in the trunk, and 27 in the extremities. Tumor stages were as follows: $\mathrm{I}=19, \mathrm{II}=13, \mathrm{III}=16$, and $4=10$. The mean (range) survival time in months after diagnosis was 91.2 years (1-204).

\section{Immunohistochemistry}

Two GRP78 immunohistochemical staining patterns were recognized within the invasive component:

Pattern 1. Fade: strong, fine cytoplasmic staining was observed in the in situ and superficial invasive component, with a progressive loss or 'fading out' of staining in the deeper invasive portions of the melanoma (34 cases, $59 \%$ of total cases, Figure 2).

Pattern 2. Regain: strong cytoplasmic staining in in situ and superficial components, with a conspicuous stain-poor central area, followed by a regain of expression at the deep invasive edge of the tumor (24 cases, $41 \%$ of total cases, Figure 3 ).

Three patterns of MTJ1 staining were observed (Figure 4): (1) negative staining of tumor cells in which no stain was observed; (2) diffuse blush/weak staining of tumor cells in which faint, cytoplasmic staining was observed; and (3) strong positive staining in which conspicuous coarse cytoplasmic staining was observed. Nuclear staining was negative.

\section{Statistics}

There was no relationship between the pattern of staining for GRP78 and the presence of metastasis $\left(P>0.3\right.$ by $\chi^{2}$-test). Figure 5 shows a Kaplan-Meier plot of overall survival versus the staining pattern for GRP78. The upper curve is for those with a fadetype pattern and the lower curve is for those with a regain pattern. Although the mean survival time for the fade group was 133 months, the mean for the regain group was 79 months, and this difference was significant by the log-rank test $(P=0.016)$. There was also a significant difference in tumor thickness in the two patterns. Although the mean thickness in the fade group was $2.1 \mathrm{~mm}$, the mean in the regain group was $3.8 \mathrm{~mm}(P=0.001$ by Kruskal-Wallis nonparametric test). Thus, the regain group comprised thicker tumors, and this may explain some of the difference in survival time. Figure 6 shows a boxplot of tumor thickness for the two patterns and shows that the fade group had thinner tumors.

Figure 7 shows a Kaplan-Meier plot of overall survival versus staining pattern for MTJ1. The uppermost curve is for those with a strong staining, the middle curve for those with negative staining, and the lowest curve for those with 'blush' staining. The mean survival times for these three 

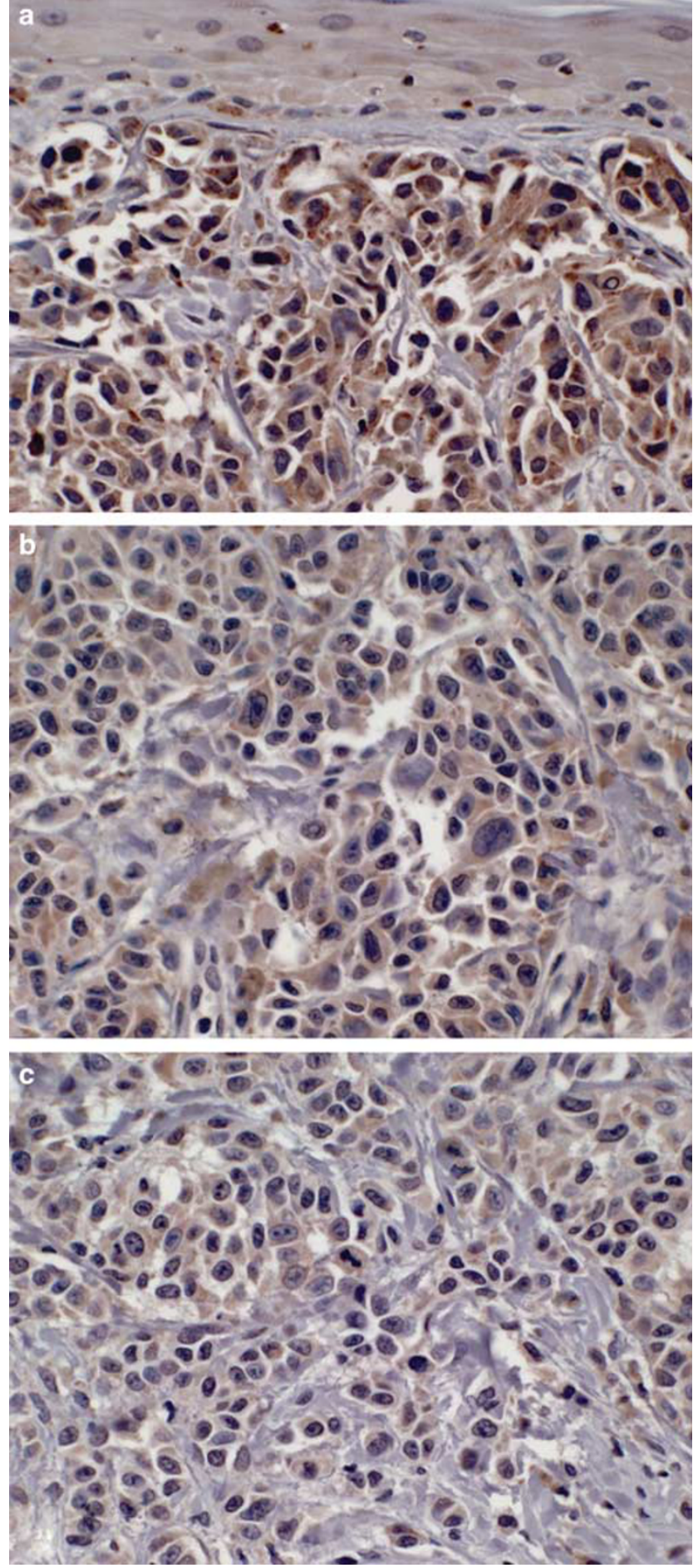

Figure 2 Fade pattern of GRP78 staining in a malignant melanoma. (a) Positive staining in the in situ and superficial component just beneath the basal layer $(\times 400)$, (b) middle portion of the tumor showing less-intense staining than regions above it $(\times 400)$, and $(\mathbf{c})$ deepest component with almost total loss of staining $(\times 400)$.

groups were 165 months, 117 months, and 75 months, respectively, and this difference was significant by the log-rank test $(P=0.002)$. Thus, 'blush' staining was the worst pattern as far as
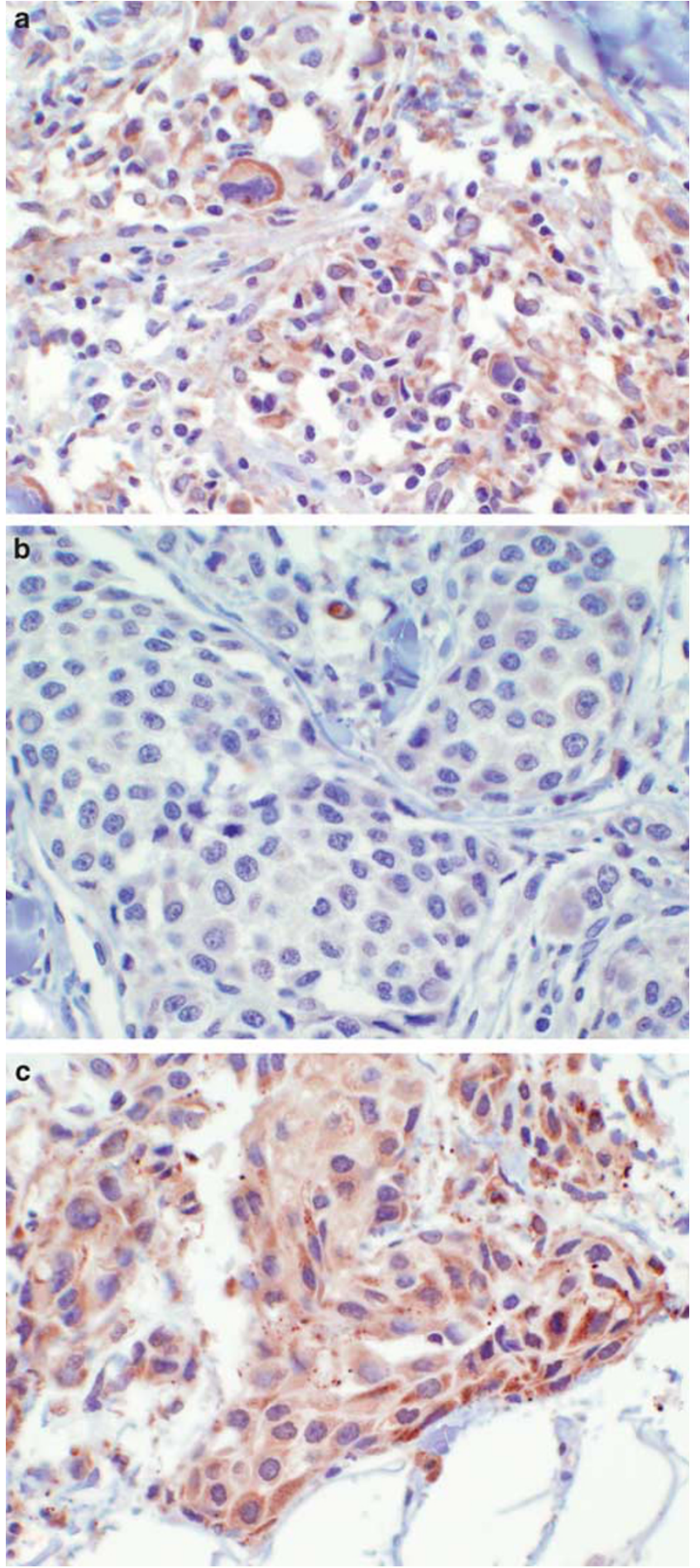

Figure 3 Regain of GRP78 expression in the deep invasive edge of a malignant melanoma. (a) Positive superficial component seen just beneath the basal layer $(\times 400)$, (b) middle portion of the tumor with loss of staining $(\times 400)$, and $(\mathbf{c})$ deep invasive edge with brisk regain of GRP78 expression $(\times 400)$.

survival was concerned. Thickness also differed among these three groups, with a mean thickness of $2.1 \mathrm{~mm}$ for the strong staining group, $2.9 \mathrm{~mm}$ for the negative group, and $3.2 \mathrm{~mm}$ for the blush group, and 

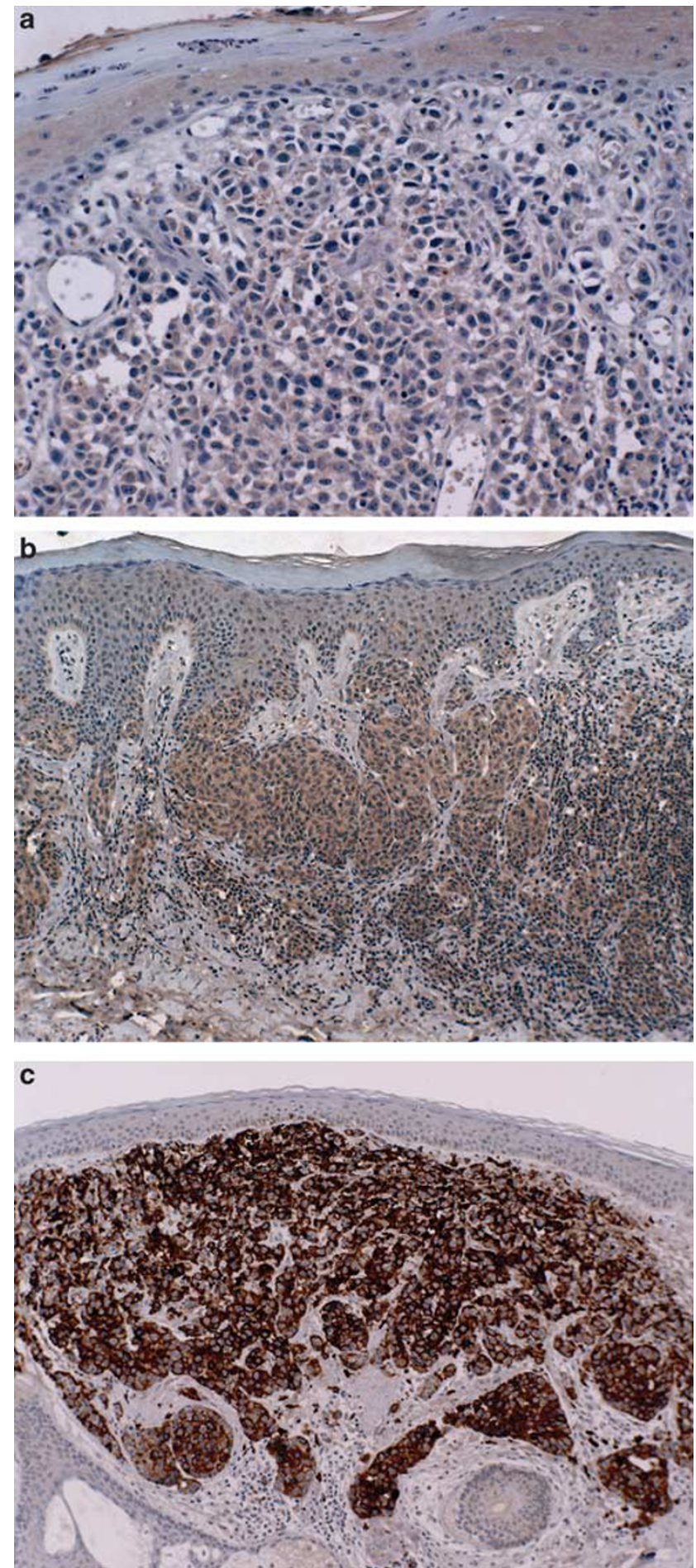

Figure 4 Spectrum of MTJ1 staining. Patients showed one of the three main patterns of MTJ1 protein expression: (a) negative $(\times 200)$, (b) weak or 'blush' staining $(\times 100)$, and $(\mathbf{c})$ strong positive $(\times 100)$.

although the blush group had the worst overall survival, the differences in thickness were not significant by the Kruskal-Wallis test $(P>0.2)$.

Finally, when comparing the pattern of GRP78 expression and MTJ1 staining, $56 \%$ of the GRP78

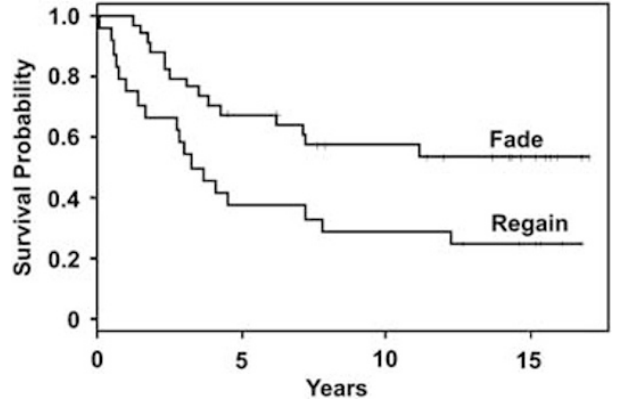

Figure 5 Kaplan-Meier Plot of overall survival versus staining pattern for GRP78. The upper curve is for melanoma cases with a fade-type pattern, and the lower curve is for those with a regain pattern. Mean survival time for the fade group was 133 months, and the mean for the regain group was 79 months (significant by log-rank test).

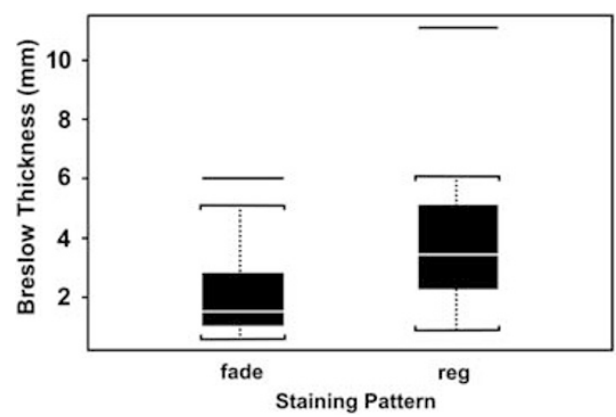

Figure 6 Tumor thickness and pattern of GRP78 staining. Mean thickness in the fade group was $2.1 \mathrm{~mm}$ and the mean in the regain group was $3.8 \mathrm{~mm}(P=0.001$ by Kruskal-Wallis nonparametric test).

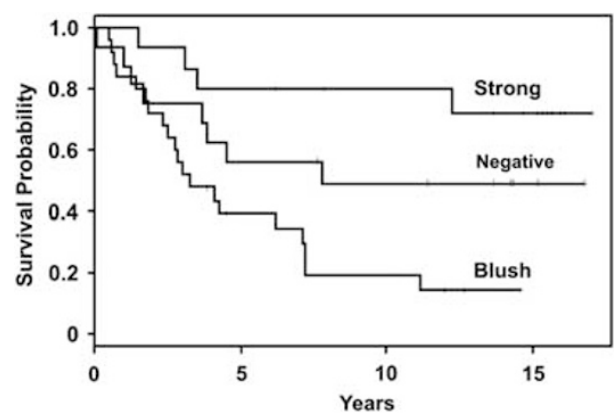

Figure 7 Overall survival versus staining pattern for MTJ1. The uppermost curve is for those with a strong staining, the middle curve for those with negative staining, and the lowest curve for those with blush staining. The mean survival times for these three groups were 165 months, 117 months, and 75 months, respectively, and this difference was significant by the log-rank test $(P=0.002)$.

regain group (deeper tumor, poorer survival) also had blush MTJ1 staining (deeper tumors, poorer survival), whereas only $36 \%$ of the GRP78 fade group (more superficial tumor, better survival) had MTJ1 blush staining; however, this difference was not significant by the $\chi^{2}$-test $(P>0.9)$. 


\section{Discussion}

Glucose-regulated proteins are ER-stationed chaperone proteins that, as a class, have been described with respect to tumor progression and metastasis. ${ }^{45}$ Of the GRP family, GRP78 is the best studied. Robust induction of GRP78 occurs in many malignancies. ${ }^{46}$ Furthermore, GRP78 has been shown to be involved in a number of physiological events that are key to tumor growth and survival. ${ }^{47,48}$ Once upregulated or transcriptionally active, GRP78 can promote tumor proliferation through a variety of mechanisms. ${ }^{49-51}$

We performed an observational investigation of GRP78 and MTJ1 expression in primary cutaneous melanoma and correlated our observations with various clinical parameters. Using a polyclonal antibody directed against an epitope in the NH2terminal domain of the GRP78 protein, immunohistochemical analysis of 58 cases of melanoma revealed two distinct patterns of expression. One pattern was marked by a strong cytoplasmic staining of the in situ and superficial invasive component, with a marked decrease in protein expression in deeper tumor levels. In this group, the regain of GRP78 expression was not observed. The second pattern of GRP78 expression was identical to the first, except for the fact that an abrupt transition to protein reexpression was observed at the invasive edge of the melanoma, leaving a conspicuous stainpoor central region within the melanoma. As a group, cases that showed a gradual loss or fade of GRP78 expression had overall more shallow depths of invasion and longer overall survival (Figure 8) when compared with the group of cases in which regain of GRP78 expression was observed, which had deeper overall tumor thickness and a decreased mean survival (Figure 9).

Using an antibody for an epitope in the MTJ1 region comprising amino acid $\mathrm{V}^{110}$ AIYEVLKD-

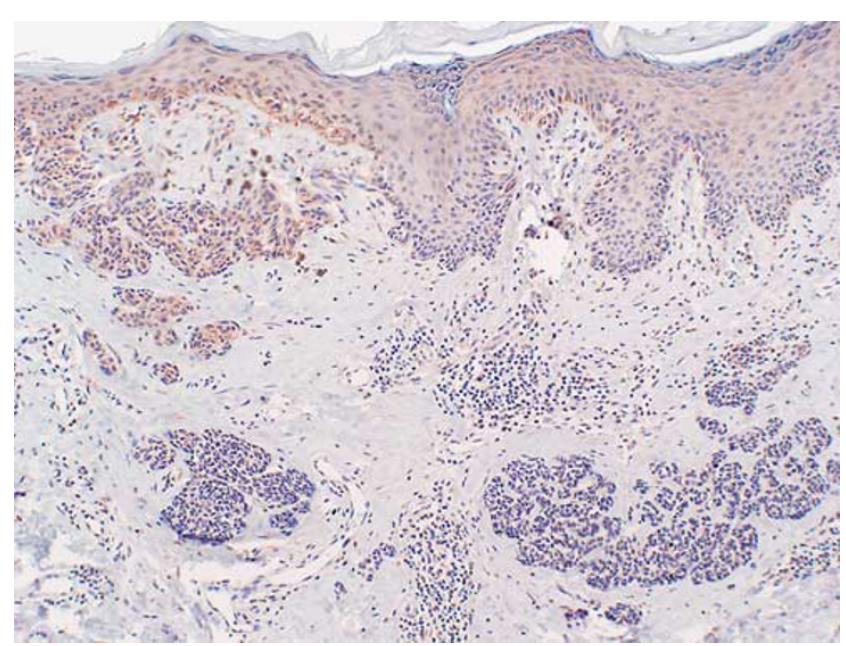

Figure 8 Summary of the GRP78 fade group. In all, 34 cases show a gradual loss or fade of the GRP78 expression pattern and have both less-invasive tumors and greater survival when compared with the regain of expression pattern.
DERRQRYDDIL ${ }^{129}$, we observed a broader spectrum of staining patterns, which we categorized as either negative, weak/blush, or strong staining. We observed that patients with weak/blush staining had worse survival, when compared with patients with negative or strong staining. The patterns of MTJ1 expression were not, however, significantly associated with any one particular pattern of GRP78 staining (regain or fade).

Similar to our observations in primary cutaneous melanoma, GRP78 expression shows both temporal and qualitative dynamism within various tumor types during tumor progression. For example, in prostate cancer, stronger GRP78 staining was associated with decreased overall survival and clinical recurrence-free survival. ${ }^{39}$ Wang et $a l^{38}$ showed increased levels of GRP78 expression in more poorly differentiated and higher-stage adenocarcinomas of the lung, with similar observations being made for esophageal adenocarcinoma. ${ }^{37}$ It is noteworthy that in a subset of cases of esophageal cancers studied, tumors either showed an increased expression of GRP78 in early- (pT1) or more advanced-stage tumors (pT3), with lower levels of expression observed in intermediate-stage lesions. These findings are analogous to our findings of a decreased GRP78 protein expression in malignant cells of more intermediate Breslow thickness and support the notion that GRP78 expression by malignant cells is not static and that expression under certain circumstances may result in different functions at different phases of tumor progression.

In light of these findings, it is important to note that these three studies, along with ours, all used the same Anti-GRP78 polyclonal antibody (Santa Cruz Biotechnology, catalog number SC-1050) against the $\mathrm{N}$-terminus portion of the protein. This

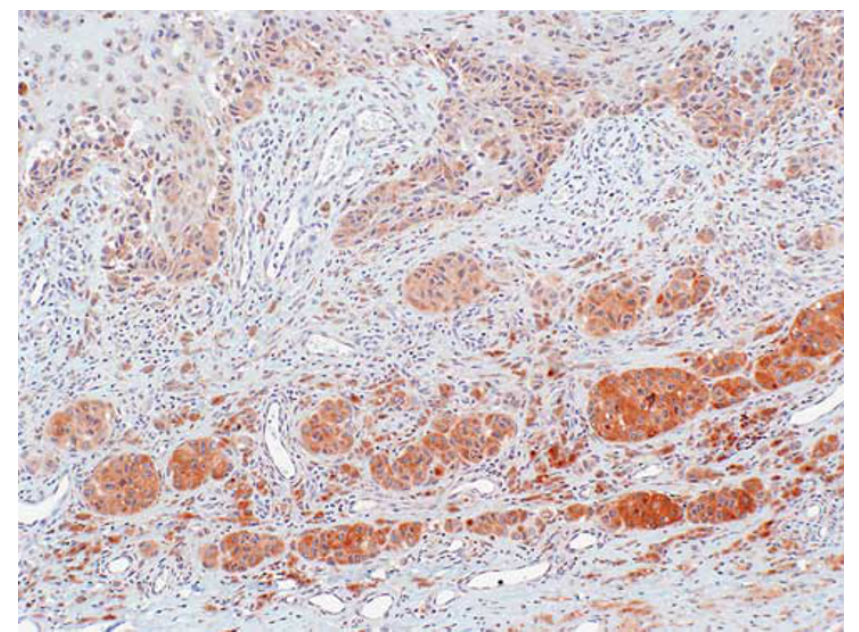

Figure 9 Summary of the GRP78 regain group. In all, 24 cases show a regain of GRP78 expression at the deep invasive component. These cases were more invasive with shorter patient survival when compared with patients with a loss of GRP78 expression. 
is important because of the recent advances made in the understanding of the relationship between GRP78 function and the domain-specific interactions that it has with proteins such as MTJ1. ${ }^{52}$ Differences in GRP78 immunohistochemical reactivity may in part be explained by conformational changes in GRP78 specific to a particular tumor type at a particular point in the progression of that tumor. With this in mind, we attempted to show a different pattern of staining (that is, loss of superficial and deep staining in the melanoma with a central stainrich region) using an anti-GRP78 antibody that we produced, directed against the COOH-terminal domain of GRP78. Our results, however, were nonspecific without the reproducible patterns seen with the use of the antibody directed against an epitope in the NH2-terminal domain (Papalas, unpublished observations). Recently, Zhuang et $a l^{40}$ showed a negative association between the overall strength of GRP78 expression in melanoma and various clinical parameters. Unlike our study, which used antibodies against the $\mathrm{N}$-terminus, they used a commercial antibody directed against the C-terminus of GRP78. Similar to our unpublished observations, they did not observe a regain of GRP78 expression, or any specific pattern of expression, when using an antibody directed against the $\mathrm{COOH}$-terminal domain. Nonetheless, their observations have emphasized the importance of GRP78 expression in melanoma progression.

Owing to the diverse functions of GRP78 described both in neoplasia and the UPR, there are several theories that may account for the regain of GRP78 seen in the deeper levels of melanomas we studied. In its traditional role as a heat shock protein of the HSP 70 family, GRP78 and other related proteins function in response to cellular stress. ${ }^{53}$ As melanomas progress and invade into deeper levels, there are concomitant changes in a number of microenvironmental physical conditions, ${ }^{54,55}$ of which hypoxia is paramount, which has been shown to trigger a number of molecular cascades in melanoma cell lines. ${ }^{56,57}$ In fact, hypoxic stress by itself leads to an increased expression of GRP78 in cultured human fibrosarcoma cells. ${ }^{58}$ Hence, solid malignancies, such as melanoma, which retain their capacity to respond to cell stressors, will upregulate the expression of GRP78. ${ }^{30}$ This is consistent with our observations of progressive loss and regain of expression of GRP78 at the deeper invasive edge of melanomas.

Glucose-regulated protein 78 is also involved in the inhibition of apoptosis. ${ }^{59}$ As local microenvironmental conditions evolve and GRP78 expression increases, melanoma cells gain resistance to intra and extracellular inducers of apoptosis. ${ }^{60}$ If increased expression of GRP78 confers resistance to apoptosis, this could help to explain why we observed a decreased overall survival in patients who showed regain of GRP78 at the invasive edge of their melanomas.
More recently, GRP78 has been characterized on cell membranes. ${ }^{61}$ Flow cytometric analysis of thapsigargin-treated rhabdomyosarcoma cells showed cell surface expression of the heat shock protein. ${ }^{62}$ Investigation into the function of cell membrane-associated GRP78 has shown its involvement in various signal transduction cascades. ${ }^{63,64}$ In sum, these pathways are progrowth and antiapoptotic. ${ }^{23-25,63,65}$ One of the limitations of our study is that differences in GRP78 cellular and subcellular distribution between superficial GRP78-positive melanoma cells and deep 'regain' GRP78-positive melanoma cells were not assessed. We did not observe membrane-only/predominant staining in our set of cases. The predominant pattern was strong cytoplasmic staining with occasional perinuclear clearing. More study is needed to address the potential localization and functional differences in GRP78 between different populations of in situ melanoma cells.

As cell surface GRP78 forms a complex with ERstationed MTJ1, ${ }^{23}$ we also hypothesized that the immunohistochemical pattern of MTJ1 expression would be similar to that observed in GRP78. The pattern of MTJ1 staining showed both qualitative and quantitative differences from the patterns we observed with GRP78, but no statistical relationship between the pattern of MTJ1 staining intensity and regain of GRP78 expression was seen. However, when considered independently, the tumors weakly expressing MTJ1 had worse overall survival $(P=0.002)$ than did tumors with a strong or negative expression. When the GRP78 regain group was compared with the fade group, $56 \%$ of fade group had tumors with weak MTJ1 staining versus $36 \%$ of the regain group, but this difference was not significant by the $\chi^{2}$-test $(P>0.9)$. In addition, when compared with melanomas with any expression of MTJ1, those with no staining had Breslow thickness (average for the group, $2.9 \mathrm{~mm}$ ) between those with strong positive $(2.1 \mathrm{~mm})$ and those with weak $(3.2 \mathrm{~mm})$ MTJ1 staining. Although these differences in tumor depth between MTJ1 groups failed to meet statistical significance (Kruskal-Wallis test ${ }^{63}$ ), they do suggest that MTJ1, similar to GRP78, is expressed differentially at various phases of melanoma invasion. Out of the entire patient set with adequate tissue left over after GRP78 IHC $(n=56), 71 \%$ of cases showed some degree of MTJ1 staining (either weak or positive). This finding, at the very least, shows that GRP78 and MTJ1 are coexpressed in almost three-fourth of the tumors that we studied.

Finally, as an exploratory study regarding the potential importance of GRP78 and MTJ1 in primary cutaneous melanoma, testing for their independent contributions to outcomes will require a follow-up study with more patients. We already demonstrated that GRP78 is not statistically independent from tumor thickness, and our results hint that MTJ1 is also related to tumor thickness. Thus, we conclude that these biological markers must be related to 
important aspects of melanoma, because thickness certainly is. Which among these is the most closely related to outcomes is for the moment uncertain.

We describe for the first time two distinct patterns of GRP78 expression within a single tumor type (primary cutaneous malignant melanoma), as well as correlate these patterns with MTJ1 expression and several clinicopathological parameters. Additional studies are needed to both clarify the role of GRP78 in malignant melanoma and further characterize its reexpression at deeper levels within invasive melanoma and its relationship to proteins, such as MTJ1, which function to augment the cell surface signaling capacity of GRP78.

\section{Acknowledgements}

We extend our special thanks to Steve Conlon of PhotoPath at Duke University Medical Center for his technical assistance in the preparation of this paper.

\section{Disclosure/conflict of interest}

The authors declare no conflict of interest.

\section{References}

1 Geller AC, Miller DR, Annas GD, et al. Melanoma incidence and mortality among US Whites, 1969-1999. JAMA 2002;288:1719-1720.

2 Garbe C, Eigentler TK. Diagnosis and treatment of cutaneous melanoma: state of the art 2006. Melanoma Res 2007;17:117-127.

3 Hocker TL, Singh MK, Tsao H. Melanoma genetics and therapeutic approaches in the 21st century: moving from the benchside to the bedside. J Invest Dermatol 2008;128:2575-2595.

4 Sekulic A, Haluska Jr P, Miller AJ, et al. Malignant melanoma in the 21st century: the emerging molecular landscape. Mayo Clin Proc 2008;83:825-846.

5 Demierre MF, Sabel MS, Margolin KA, et al. State of the science 60th anniversary review: 60 years of advances in cutaneous melanoma epidemiology, diagnosis, and treatment, as reported in the journal Cancer. Cancer 2008;113:1728-1743.

6 Roesch A, Becker B, Meyer S, et al. Overexpression and hyperphosphorylation of retinoblastoma protein in the progression of malignant melanoma. Mod Pathol 2004;18:565-572.

7 Li LX, Crotty KA, McCarthy SW, et al. A zonal comparison of MIB1-Ki67 immunoreactivity in benign and malignant melanocytic lesions. Am J Dermatopathol 2000;22:489-495.

8 Li LX, Crotty KA, Scolyer RA, et al. Use of multiple cytometric markers improves discrimination between benign and malignant melanocytic lesions: a study of DNA microdensitometry, karyometry, argyrophilic staining of nucleolar organizer regions and MIB1-Ki67 immunoreactivity. Melanoma Res 2003;13: 581-586.
9 Shiu RP, Pouyssegur J, Pastan I. Glucose depletion accounts for the induction of two transformation sensitive membrane proteins in Rous Sarcoma virustransformed chick embryo fibroblasts. Proc Natl Acad Sci USA 1977;74:3840-3844.

10 Lee AS. The glucose-regulated proteins: stress induction and clinical applications. Trends Biochem Sci 2001;26:504-510.

11 Schroder M, Kaufman RJ. The mammalian unfolded protein response. Annu Rev Biochem 2005;74: 739-789.

12 Awe K, Lambert C, Prange R. Mammalian BiP controls posttranslational ER translocation of the hepatitis B virus large envelope protein. FEBS Lett 2008;582: 3179-3184.

$13 \mathrm{Li} \mathrm{J}$, Lee B, Lee AS. Endoplasmic reticulum stress induced apoptosis: multiple pathways and activation of p53-up-regulated modulator of apoptosis (PUMA) and NOXA by p53. J Biol Chem 2006;281: 7260-7270.

14 Reddy RK, Mao C, Baumeister P, et al. Endoplasmic reticulum chaperone protein GRP78 protects cells from apoptosis induced by topoisomerase inhibitors. J Biochem Chem 2003;278:20915-20924.

15 Liu Y, Steiniger SCJ, Kim YS, et al. Mechanistic studies of a peptidic GRP78 ligand for cancer cell-specific drug delivery. Mol Pharmacol 2007;4:435-447.

16 Delpino A, Castelli M. The $78 \mathrm{kDa}$ glucose-regulated protein (GRP78/BIP) is expressed on the cell membrane, is released into cell culture medium and is also present in human peripheral circulation. Biosci Rep 2002;22:407-420.

17 Misra UK, Gonzalez-Gronow M, Gawdi G, et al. A novel receptor function for the heat shock protein Grp78: silencing of Grp78 gene expression attenuates alpha2M*-induced signalling. Cell Signal 2004;16: 929-938.

18 Shen X, Zhang K, Kaufman RJ. The unfolded protein response-a stress signaling pathway of the endoplasmic reticulum. J Chem Neuroanat 2004;28: 79-92.

19 Zhang K, Kaufman RJ. The unfolded protein response: a stress signaling pathway critical for health and disease. Neurology 2006;66:S102-S109.

20 Mintz PJ, Kim J, Do KA, et al. Fingerprinting the circulating repertoire of antibodies from cancer patients. Nat Biotechnol 2003;21:57-63.

21 Chinni SR, Falchetto R, Gercel-Taylor C, et al. Humoral immune responses to cathepsin $\mathrm{D}$ and glucose-regulated protein 78 in ovarian cancer patients. Clin Cancer Res 1997;3:1557-1564.

22 Rauschert N, Brandlein S, Holzinger E, et al. A new tumor-specific variant of GRP78 as target for antibodybased therapy. Lab Invest 2008;88:375-386.

23 Misra UK, Gonzalez-Gronow M, Gawdi G, et al. The role of MTJ-1 in cell surface translocation of GRP78, a receptor for alpha 2-macroglobulin-dependent signaling. J Immunol 2005;174:2092-2097.

24 Misra UK, Pizzo SV. Heterotrimeric Galphaq11 coimmunoprecipitates with surface-anchored GRP78 from plasma membranes of alpha2 $\mathrm{M}^{*}$-stimulated macrophages. J Cell Biochem 2008;104:96-104.

25 Xie P, Browning DD, Hay N, et al. Activation of NFkappa B by bradykinin through a Galpha(q)- and Gbeta gamma-dependent pathway that involves phosphoinositide 3-kinase and Akt. J Biol Chem 2000;275: 24907-24914. 
26 Kaufman RJ. Orchestrating the unfolded protein response in health and disease. J Clin Invest 2002;110:1389-1398.

27 Chevalier M, Rhee H, Elguindi EC, et al. Interaction of murine BiP/GRP78 with the DnaJ homologue MTJ1. J Biol Chem 2000;275:19620-19627.

28 Kroczynska B, King-Simmons L, Alloza L, et al. BIP cochaperone MTJ1/ERDJ1 interacts with inter-alphatrypsin inhibitor heavy chain 4. Biochem Biophys Res Commun 2005;338:1467-1477.

29 Brightman SE, Blatch GL, Zetter BR. Isolation of a mouse cDNA encoding MTJ1, a new murine member of the DnaJ family of proteins. Gene 1995;153: 249-254.

30 Lee AS. GRP78 induction in cancer: therapeutic and prognostic implications. Cancer Res 2007;67: 3496-3499.

31 Miyake H, Hara I, Arakawa S, et al. Stress protein GRP78 prevents apoptosis induced by calcium ionophore, ionomycin, but not by glycosylation inhibitor, tunicamycin, in human prostate cancer cells. J Cell Biochem 2000;77:396-408.

32 Qian Y, Harris ED, Zheng Y, et al. Lead targets GRP78, a molecular chaperone, in C6 rat glioma cells. Toxicol Appl Pharmacol 2000;163:260-266.

33 Gazit G, Lu J, Lee AS. De-regulation of GRP stress protein expression in human breast cancer cell lines. Breast Cancer Res Treat 1999;54:135-146.

34 Tang D, Khaleque MA, Jones EL, et al. Expression of heat shock proteins and heat shock protein messenger ribonucleic acid in human prostate carcinoma in vitro and in tumors in vivo. Cell Stress Chaperones 2005;10:46-58.

35 Luk JM, Lam CT, Siu AF, et al. Proteomic profiling of hepatocellular carcinoma in Chinese cohort reveals heat-shock proteins (Hsp27, Hsp70, GRP78) up-regulation and their associated prognostic values. Proteomics 2006;6:1049-1057.

36 Zhang J, Jiang Y, Jia Z, et al. Association of elevated GRP78 expression with increased lymph node metastasis and poor prognosis in patients with gastric cancer. Clin Exp Metastasis 2006;23:401-410.

37 Langer R, Feith M, Siewert JR, et al. Expression and clinical significance of glucose regulated proteins GRP78 (BiP) and GRP94 (GP96) in human adenocarcinomas of the esophagus. BMC Cancer 2008;8:70.

38 Wang Q, He Z, Zhang J, et al. Overexpression of endoplasmic reticulum molecular chaperone GRP94 and GRP78 in human lung cancer tissues and its significance. Cancer Detect Prev 2005;29:544-551.

39 Daneshmand S, Quek ML, Lin E, et al. Glucoseregulated protein GRP78 is up-regulated in prostate cancer and correlates with recurrence and survival. Hum Pathol 2007;38:1547-1552.

40 Zhuang L, Scolyer RA, Lee CS, et al. Expression of glucose-regulated stress protein GRP78 is related to progression of melanoma. Histopathology 2009;54: $462-470$.

41 Kim Y, Lillo AM, Steiniger SC, et al. Targeting heat shock proteins on cancer cells: selection, characterization, and cell-penetrating properties of a peptidic GRP78 ligand. Biochemistry 2006;45:9434-9444.

42 Gupta P, Walter MR, Su ZZ, et al. BiP/GRP78 is an intracellular target for MDA-7/IL-24 induction of cancer-specific apoptosis. Cancer Res 2006;66: 8182-8191.
43 Gupta P, Su ZZ, Lebedeva IV, et al. mda-7/IL-24: multifunctional cancer-specific apoptosis-inducing cytokine. Pharmacol Ther 2006;111:596-628.

44 Nonaka D, Chiriboga L, Rubin BP. Differential expression of S100 protein subtypes in malignant melanoma, and benign and malignant peripheral nerve sheath tumors. J Cutan Pathol 2008;35:1014-1019.

$45 \mathrm{Fu} \mathrm{Y}$, Lee AS. Glucose regulated proteins in cancer progression, drug resistance and immunotherapy. Cancer Biol Ther 2006;5:741-744.

46 Dong D, Dubeau L, Bading J, et al. Spontaneous and controllable activation of suicide gene expression driven by the stress-inducible Grp78 promoter resulting in eradication of sizable human tumors. Hum Gene Ther 2004;15:553-561.

47 Dong D, Ni M, Li J, et al. Critical role of the stress chaperone GRP78/BiP in tumor proliferation, survival, and tumor angiogenesis in transgene-induced mammary tumor development. Cancer Res 2008;68:498-505.

$48 \mathrm{Li} \mathrm{J}$, Lee AS. Stress induction of GRP78/BiP and its role in cancer. Curr Mol Med 2006;6:45-54.

49 Chang SC, Erwin AE, Lee AS. Glucose-regulated protein (GRP94 and GRP78) genes share common regulatory domains and are coordinately regulated by common trans-acting factors. Mol Cell Biol 1989;9: 2153-2162.

50 Ramsay RG, Ciznadija D, Mantamadiotis T, et al. Expression of stress response protein glucose regulated protein-78 mediated by c-Myb. Int J Biochem Cell Biol 2005;37:1254-1268.

51 Misra UK, Deedwania R, Pizzo SV. Activation and cross-talk between Akt, NF-\{kappa\}B, and unfolded protein response signaling in 1-LN prostate cancer cells consequent to ligation of cell surface-associated GRP78. J Biol Chem 2006;281:13694-13707.

52 Awad W, Estrada I, Shen Y, et al. BiP mutants that are unable to interact with endoplasmic reticulum DnaJ proteins provide insights into interdomain interactions in BiP. Proc Natl Acad Sci USA 2008;105: 1164-1169.

53 Macario AJL, De Macario EC. Sick chaperones, cellular stress, and disease. N Engl J Med 2005;353:1489-1501.

54 Spinella F, Rosano L, Di Castro V, et al. Endothelin-1 and endothelin-3 promote invasive behavior via hypoxia-inducible factor-1 $\{$ alpha\} in human melanoma cells. Cancer Res 2007;67:1725-1734.

55 Sun B, Zhang S, Zhang D, et al. The influence of different microenvironments on melanoma invasiveness and microcirculation patterns: an animal experiment study in the mouse model. J Cancer Res Clin Oncol 2007;133:979-985.

56 Yamashita T, Ohneda K, Nagano M, et al. Hypoxiainducible transcription factor-2alpha in endothelial cells regulates tumor neovascularization through activation of ephrin A1. J Biol Chem 2008;283: 18926-18936.

57 Bedogni B, Warneke JA, Nickoloff BJ, et al. Notch1 is an effector of Akt and hypoxia in melanoma development. J Clin Invest 2008;118:3660-3670.

58 Davidson DJ, Haskell C, Majest S, et al. Kringle 5 of human plasminogen induces apoptosis of endothelial and tumor cells through surface-expressed glucoseregulated protein 78. Cancer Res 2005;65:4663-4672.

59 Shu CW, Sun FC, Cho JH, et al. GRP78 and Raf-1 cooperatively confer resistance to endoplasmic reticulum stress-induced apoptosis. J Cell Physiol 2008;215: $627-635$ 
60 Jiang CC, Chen LH, Gillespie S, et al. Inhibition of MEK sensitizes human melanoma cells to endoplasmic reticulum stress-induced apoptosis. Cancer Res 2007; 67:9750-9761.

61 Shin BK, Wang H, Yim AM, et al. Global profiling of the cell surface proteome of cancer cells uncovers an abundance of proteins with chaperone function. J Biol Chem 2003;278:7607-7616.

62 Delpino A, Piselli P, Vismara D, et al. Cell surface localization of the $78 \mathrm{kD}$ glucose regulated protein (GRP 78) induced by thapsigargin. Mol Membr Biol 1998;15:21-26.

63 Misra UK, Gonzalez-Gronow M, Gawdi G, et al. The Role of Grp 78 in alpha 2-macroglobulin-induced signal transduction. Evidence from RNA interference that the low density lipoprotein receptor-related protein is associated with, but not necessary for, GRP 78-mediated signal transduction. J Biol Chem 2002; 277:42082-42087.

64 Shani G, Fischer WH, Justice NJ, et al. GRP78 and Cripto form a complex at the cell surface and collaborate to inhibit transforming growth factor signaling and enhance cell growth. Mol Cell Biol 2008;28:666-677.

65 Gonzalez-Gronow M, Cuchacovich M, Llanos C, et al. Prostate cancer cell proliferation in vitro is modulated by antibodies against glucose-regulated protein 78 isolated from patient serum. Cancer Res 2006;66: 11424-11431. 